\title{
Validation of Alternating Kernel Mixture Method: Application to Tissue Segmentation of Cortical and Subcortical Structures
}

\author{
Nayoung A. Lee, Carey E. Priebe, Michael I. Miller, and J. Tilak Ratnanather \\ Center for Imaging Science, Johns Hopkins University, Baltimore, MD 21218, USA \\ Correspondence should be addressed to Nayoung A. Lee, nayoung@cis.jhu.edu
}

Received 4 September 2007; Revised 28 February 2008; Accepted 25 June 2008

Recommended by Daniel Howard

This paper describes the application of the alternating Kernel mixture (AKM) segmentation algorithm to high resolution MRI subvolumes acquired from a $1.5 \mathrm{~T}$ scanner (hippocampus, $n=10$ and prefrontal cortex, $n=9$ ) and a $3 \mathrm{~T}$ scanner (hippocampus, $n=10$ and occipital lobe, $n=10$ ). Segmentation of the subvolumes into cerebrospinal fluid, gray matter, and white matter tissue is validated by comparison with manual segmentation. When compared with other segmentation methods that use traditional Bayesian segmentation, AKM yields smaller errors $(P<.005$, exact Wilcoxon signed rank test $)$ demonstrating the robustness and wide applicability of AKM across different structures. By generating multiple mixtures for each tissue compartment, AKM mimics the increased variation of manual segmentation in partial volumes due to the highly folded tissues. AKM's superior performance makes it useful for tissue segmentation of subcortical and cortical structures in large-scale neuroimaging studies.

Copyright (c) 2008 Nayoung A. Lee et al. This is an open access article distributed under the Creative Commons Attribution License, which permits unrestricted use, distribution, and reproduction in any medium, provided the original work is properly cited.

\section{INTRODUCTION}

Current magnetic resonance image (MRI) studies investigate abnormalities of cortical and subcortical structures in neurodevelopmental and neurodegenerative disorders. These studies require a delineation of a region of interest (ROI) by manual segmentation by an expert rater. For example, studies on Alzheimer's disease and mild cognitive impairment examine the hippocampus [1] while those in schizophrenia have studied the occipital lobe and prefrontal cortex $[2,3]$. Once the ROI is defined, segmentation into tissue types such as gray matter $(\mathrm{GM})$, white matter (WM), or cerebrospinal fluid (CSF) can assess subtle volume changes caused by disease $[4,5]$. While manual segmentation would provide gold standard, it is labor intensive limiting the number of subjects in any study [6]. Also, the rater needs to be trained to ensure small inter- or intrarater variation. Therefore, it is necessary to develop a method that allows for efficient processing of large number of subjects with high interor intrarater reliability, thereby increasing statistical power. Such a method will facilitate greater understanding of shape change in networks of cortical structures implicated in neuropsychiatric diseases $[7,8]$.
A variety of methods have been proposed for the segmentation of subcortical tissue such as the hippocampus [911] and cortical tissues such as prefrontal cortex, cingulate cortex, and planum temporale [12-16]. However, even though tissue classification methods have been improving in their performance, relatively low accuracy (comparing with expert-rater standards) has prevented accurate structural segmentation, for example, distinguishing the hippocampus from surrounding structures in the medial temporal lobe.

Partial volume voxels containing multiple tissue types present challenges to traditional Bayesian tissue classification methods [17-24] that model each tissue type as a fixedbandwidth, single Gaussian in mixture-of-Gaussian models. Priebe et al. [25] proposed an alternating Kernel mixture (AKM) method which allowed for the flexibility of a Gaussian mixture model, with bandwidth, and the number of Gaussians selected adaptively from the data for each tissue type. The purpose of our study was to compare the performance of AKM and traditional Bayesian methods. The two methods were compared by determining which method was closer to the manual segmentation (ground truth) of cortical and subcortical structures in MRI subvolumes acquired from $1.5 \mathrm{~T}$ and $3 \mathrm{~T}$ scanners. 
The manuscript is organized as follows. Section 2 describes the AKM mixture modeling methodology in detail and other Bayesian-based segmentation methods. Section 3 describes the dataset being investigated and image analysis employed. Section 4 reports the results.

\section{METHOD}

\subsection{Alternating Kernel mixture method}

Priebe and Marchette [26] and James et al. [27] introduced a semiparametric solution to the problem of estimating the common probability density function for multiple identically distributed random variables. Their solution is an iterative one that combines parametric and nonparametric estimates with a resulting model that incorporates both the complexity and the smoothness of the data.

We applied this method to the problem of MR segmentation. Gaussian mixture modeling is a popular segmentation technique. The marginal probability density function for the observations is

$$
f=\sum_{c \in C} \pi_{c} f_{c},
$$

where $C:=\{C, G, W\}$ is the set of tissue types (CSF, GM, and $\mathrm{WM}), f_{c}$ are the class-conditional marginals, and $\pi_{c}$ are class-conditional mixing coefficients. These coefficients are nonnegative and sum to unity. Thus, the image is the sum of the three tissue types. Each class-conditional marginal is a mixture of normals given by

$$
f_{c}=\sum_{t=1}^{k_{c}} \pi_{c t} \varphi_{c t}
$$

where $\pi_{c t}$ are the strictly positive, class-specific mixing coefficients, which sum to one, and $\varphi_{c t}$ are the Gaussian probabilities with a mean of $\mu_{c t}$ and a variance of $\sigma_{c t}^{2}$. Combining these equations we see that the marginals are given by

$$
f=\sum_{c \in C} \pi_{c} \sum_{t=1}^{k_{c}} \pi_{c t} \varphi_{c t}
$$

The method estimates the class-conditional mixture complexities $k_{c}$, the mixing coefficients $\pi_{c}$, and the mixture components $\varphi_{c t}$. The Expectation-Maximization (EM) algorithm is used to estimate the means and variances of the components $[18,19]$. The mixture complexities are estimated from the data.

The method alternates between parametric finite mixture estimates and nonparametric Kernel estimates. Each estimate is based on the previous one of the opposite type. The first step of the algorithm is to find a parametric estimate and a nonparametric estimate of the data. Then, at each iteration, a parametric estimate that minimizes the distance between the two previous estimates is computed. Using the parameter estimates thus derived, a nonparametric estimate is found. This continues until the distance between two consecutive parametric estimates is smaller than a desired constant.
TABLE 1: Voxel classification based on likelihood ratio test.

\begin{tabular}{ll}
\hline Case & Classification \\
\hline$r_{1}(x)>1$ & Voxel labeled $C$ \\
$r_{2}(x)<1$ & Voxel labeled $W$ \\
$r_{1}(x)<1$ and $r_{2}(x)>1$ & Voxel labeled $G$ \\
$r_{1}(x)>1$ and $r_{2}(x)<1$ & Should not occur \\
Tie & Determined arbitrarily \\
\hline
\end{tabular}

The filtered Kernel estimate (i.e., the nonparametric estimate), with bandwidth $b$, is

$$
\tilde{f}(x ; X)=\frac{1}{n} \sum_{i=1}^{n} \sum_{t=1}^{k} \frac{\pi_{t} \varphi_{t}\left(X_{i}\right)}{f\left(X_{i}\right) b \sigma_{t}} \varphi_{0}\left(\frac{x-X_{i}}{b \sigma_{t}}\right),
$$

where $X=\left\{X_{1}, \ldots, X_{n}\right\}$ is the subject's MR voxel observation, $\sigma_{t}^{2}$ is the variance of the $t$ th component of the mixture, and $\varphi_{0}$ is the standard normal with zero mean and unit variance. The nonparametric estimates are each based on the parametric estimate from the previous iteration and are given by

$$
\hat{f}^{k}=\arg \min _{f \in F^{k}}\left\|f-\tilde{f}^{k-1}\right\|_{2}^{2}
$$

where $F^{k}$ is the class of $k$-component Gaussian mixtures, and

$$
\|f-g\|_{2}^{2}:=\int_{-\infty}^{\infty}(f(x)-g(x))^{2} d x
$$

is the integrated squared error.

To actually classify voxels, the Bayes plug-in classifier is used:

$$
g(x)=\arg \max _{c \in C} \pi_{c} f_{c}(x),
$$

where $x$ is the voxel to be labeled. The label is assigned to a class based on which one maximizes posterior probability of class membership. This can also be seen as a likelihood ratio test procedure given by

$$
\begin{gathered}
\operatorname{LRT}_{C / G(x)}=\frac{\pi_{C} f_{C}(x)}{\pi_{G} f_{G}(x)}=: r_{1}(x), \\
\mathrm{LRT}_{G / W(x)}=\frac{\pi_{G} f_{G}(x)}{\pi_{W} f_{W}(x)}=: r_{2}(x) .
\end{gathered}
$$

Tissues are then classified according to Table 1.

This method results in the voxels being classified into three categories. Priebe et al. [25] showed how a training set could be used to determine the number of components for each tissue. However, the focus of this paper is on how this could be done on a case-by-case basis using visual inspection. It was found that two or three components of CSF, GM, and WM produced the best result; in a couple of cases the complexity was better modeled with four components. 


\subsection{Bayesian segmentation}

For comparison, voxels are classified into three tissue types by Bayesian segmentation:

$$
p\left(I_{n} \mid \mu_{n}\left(h_{n}\right), \sigma_{n}^{2}\left(h_{n}\right)\right)=\prod_{n=1}^{N} \frac{1}{\sqrt{2 \pi \sigma_{n}^{2}\left(h_{n}\right)}} e^{\left(-\left(I_{n}-\mu_{n}\left(h_{n}\right)\right)^{2} / 2 \sigma_{n}^{2}\left(h_{n}\right)\right)},
$$

where $I_{n}$ is the image intensity, $h_{n}$ is the anatomical label, $\mu_{n}$ is the mean, and $\sigma_{n}^{2}$ is the variance of the Gaussian density. The algorithm is

$$
\begin{aligned}
h_{n}=\arg \max _{h_{n} \in H} \sum_{n=1}^{N}( & -\frac{1}{2} \log 2 \pi \sigma_{n}^{2}\left(h_{n}\right)-\frac{1}{2} \frac{\left(I_{n}-\mu_{n}\left(h_{n}\right)\right)^{2}}{\sigma_{n}^{2}\left(h_{n}\right)} \\
& \left.+\log \pi\left(h_{n}\right)\right),
\end{aligned}
$$

where $\pi\left(h_{n}\right)$ is the prior distribution that represents the relative amount of each of the tissue types and $H$ := $\{C, G, W\}$. As with AKM, the EM algorithm is used to estimate the means and variances of the three tissues $[18,19]$.

\subsection{Neyman-Pearson recalibration}

Bayesian segmentation can be extended to two additional classes for $C / G$ and $G / W$ partial volumes which are optimally determined by [28]

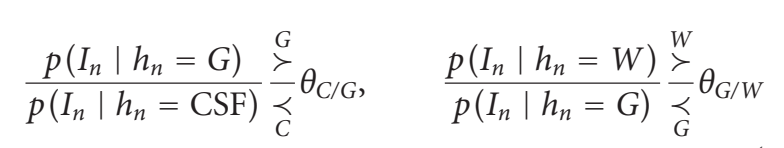

at each voxel. Here, the four thresholds $\left(\theta_{1}, \ldots, \theta_{4}\right)$ are determined by the five Gaussians. Thresholds are selected to minimize the misclassification error (Section 3.5) such that $\theta_{C / G}=\theta_{1}+t_{C / G}\left(\theta_{2}-\theta_{1}\right)$ and $\theta_{G / W}=\theta_{3}+t_{G / W}\left(\theta_{4}-\theta_{3}\right)$, where $t_{C / G} \in[0,1]$ and $t_{G / W} \in[0,1]$. The means then are used to recalibrate the segmentations yielding new thresholds. This is referred to as Neyman-Pearson recalibration.

\section{VALIDATION}

\subsection{Data acquisition}

Four different sets of ROIs were extracted from subjects scanned via the magnetization prepared rapid gradient echo sequence on different scanners. Two came from a $3 \mathrm{~T}$ scanner (10 hippocampi [29] and 5 pairs of left and right occipital lobes [30]); two came from a $1.5 \mathrm{~T}$ scanner (10 hippocampi [31] and 9 prefrontal cortices [31]). Processed datasets were reformatted to 8 bit and interpolated to $1 \times$ $1 \times 1 \mathrm{~mm}^{3}$ isotropic voxels except for the prefrontal set with

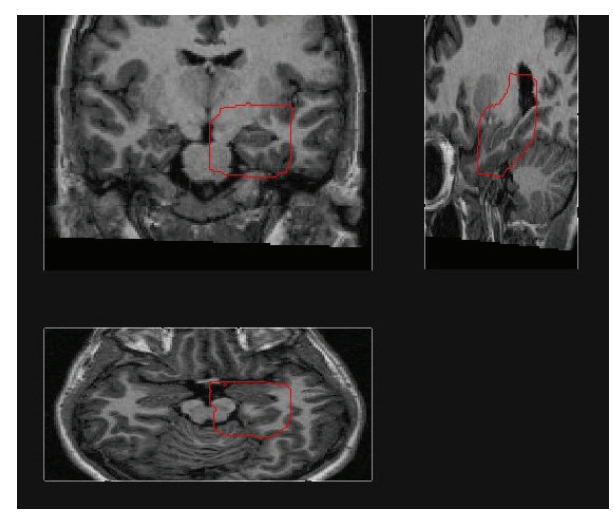

FIgURE 1: Hippocampus ROI mask delineated in red.

a resolution of $0.5 \times 0.5 \times 0.5 \mathrm{~mm}^{3}$, and are available at http://www.cis.jhu.edu/data.sets/index.html.

\subsection{MRI subvolumes}

To obtain a smaller ROI around a hippocampus, we manually outlined hippocampus and dilated it by $3 \times 3 \times 3 \mathrm{~mm}^{3}$ cubes with three iterations to generate a mask via BLOX (http://sourceforge.net/projects/blox/). Figure 1 shows an example of the mask generated for a left hippocampus. The prefrontal cortex [31] and occipital lobe [30] subvolumes were defined by an expert neuroanatomist.

\subsection{Manual tissue segmentation}

The 39 subvolumes were hand segmented into CSF, GM, and WM tissue compartments by three different raters in independent studies (e.g., $[30,31]$ ) and blind to the autosegmentation. Segmentation was done by visual inspection on contiguous sagittal slices on Analyze software [32] and saved as Analyze image data with labels for CSF, GM, and WM.

\subsection{Automated tissue segmentation}

AKM and Bayesian segmentation were applied to the 39 subvolumes. For comparison, FreeSurfer [33] and BrainVoyager [34] were used to segment the hippocampi and occipital lobes, respectively. Neyman-Pearson segmentation was applied to prefrontal cortex. The EM algorithm [18] ensured that computations were done in real time.

The 10 hippocampus subvolumes from the $1.5 \mathrm{~T}$ scanner were processed by FreeSurfer [33] to segment and label the volume by its anatomical structure. Each voxel was classified by a given anatomical label (i.e., hippocampus, ventricles). Then we group the structures into WM, GM, and CSF to create WM, GM, and CSF masks. Lateral ventricle and left inferior lateral ventricle were categorized as CSF. Cerebellum-exterior, hippocampus and amygdala were grouped as GM and cerebral white matter, thalamus proper, putamen, ventral diencephalon, and WM hypointensities were grouped as WM. 
TABLE 2: Classification error for Bayesian and AKM for hippocampi (3T).

\begin{tabular}{lcc}
\hline & Bayesian & AKM \\
\hline 1 & 0.106 & 0.099 \\
2 & 0.124 & 0.107 \\
3 & 0.234 & 0.183 \\
4 & 0.133 & 0.120 \\
5 & 0.283 & 0.114 \\
6 & 0.186 & 0.103 \\
7 & 0.153 & 0.131 \\
8 & 0.133 & 0.126 \\
9 & 0.273 & 0.172 \\
10 & 0.163 & 0.153 \\
\hline
\end{tabular}

TAble 3: Classification error for Bayesian, AKM, and NeymanPearson for prefrontal cortices (1.5T).

\begin{tabular}{lccc}
\hline & Bayesian & Neyman-Pearson & AKM \\
\hline 1 & 0.138 & 0.144 & 0.093 \\
2 & 0.103 & 0.103 & 0.101 \\
3 & 0.087 & 0.088 & 0.081 \\
4 & 0.091 & 0.097 & 0.081 \\
5 & 0.135 & 0.135 & 0.097 \\
6 & 0.093 & 0.098 & 0.088 \\
7 & 0.095 & 0.103 & 0.093 \\
8 & 0.127 & 0.127 & 0.106 \\
9 & 0.091 & 0.091 & 0.085 \\
\hline
\end{tabular}

\subsection{Quantification of segmentation accuracy}

Segmentations were compared via the $L_{1}$ distance between two distributions as a measure of misclassification error. A cost is assigned to each labeled voxel. If it was labeled correctly, that cost is 0 , and if labeled incorrectly, that cost is generally 1 . This cost, called the $L_{1}$ distance, is

$$
L_{1}=\frac{1}{2 N} \sum_{n=1}^{N} \sum_{i=1}^{m}\left|p^{A}\left(h_{n}=H_{i} \mid I_{n}\right)-p^{M}\left(h_{n}=H_{i} \mid I_{n}\right)\right|,
$$

where $p^{A}\left(h_{n} \mid I_{n}\right)$ is the posteriori probability of hypothesis $h_{n}$ at voxel $n$ for the automated, $p^{M}\left(h_{n} \mid I_{n}\right)$ is the same for the manual segmentation, and $m$ is the number of tissue types $[18,19,28,35]$. The distance measures agreement between segmentations based on distance between probability distributions [36]. The standard overlap measures penalize small objects assuming that most of the error occurs at the boundary of objects thus $L_{1}$ distance is more appropriate for assessing 3D segmentation [37]. Another standard measure, the Dice measure, was also used [38].

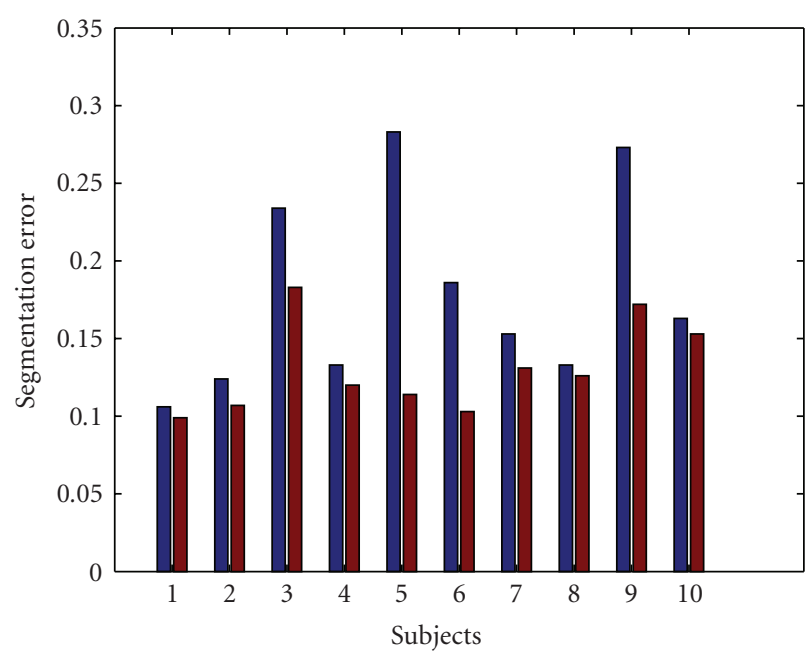

$$
\begin{aligned}
& \text { - Bayesian } \\
& \text { - AKM }
\end{aligned}
$$

Figure 2: Classification error for Bayesian (blue) and AKM (red) for hippocampi (3T).

TABLE 4: Classification error for Bayesian, BrainVoyager, and AKM for occipital lobes (3T).

\begin{tabular}{lccc}
\hline & Bayesian & BrainVoyager & AKM \\
\hline 1 & 0.170 & 0.199 & 0.119 \\
2 & 0.149 & 0.202 & 0.093 \\
3 & 0.243 & 0.221 & 0.099 \\
4 & 0.210 & 0.202 & 0.096 \\
5 & 0.236 & 0.244 & 0.112 \\
6 & 0.224 & 0.237 & 0.128 \\
7 & 0.165 & 0.111 & 0.099 \\
8 & 0.224 & 0.117 & 0.104 \\
9 & 0.157 & 0.248 & 0.119 \\
10 & 0.146 & 0.237 & 0.121 \\
\hline
\end{tabular}

TABle 5: Classification error for Bayesian, FreeSurfer and AKM for ten hippocampi (1.5T).

\begin{tabular}{lccc}
\hline & Bayesian & FreeSurfer & AKM \\
\hline 1 & 0.121 & 0.145 & 0.113 \\
2 & 0.162 & 0.225 & 0.161 \\
3 & 0.110 & 0.144 & 0.096 \\
4 & 0.131 & 0.178 & 0.109 \\
5 & 0.175 & 0.191 & 0.146 \\
6 & 0.129 & 0.169 & 0.119 \\
7 & 0.121 & 0.190 & 0.121 \\
8 & 0.121 & 0.165 & 0.121 \\
9 & 0.155 & 0.196 & 0.139 \\
10 & 0.128 & 0.143 & 0.120 \\
\hline
\end{tabular}

\section{RESULTS}

Tables 2, 3, 4, and 5 and Figures 2, 3, 4, and 5 show that $L_{1}$ distances for AKM method are lower than those 


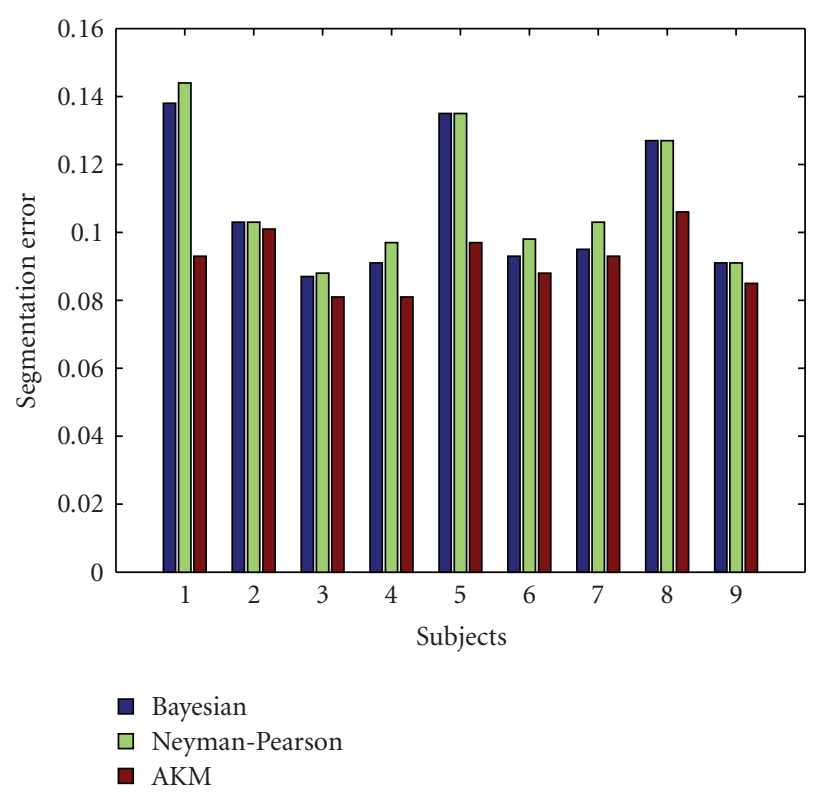

Figure 3: Classification error for Bayesian (blue), Neyman-Pearson (green), and AKM (red) for prefrontal cortices (1.5T).

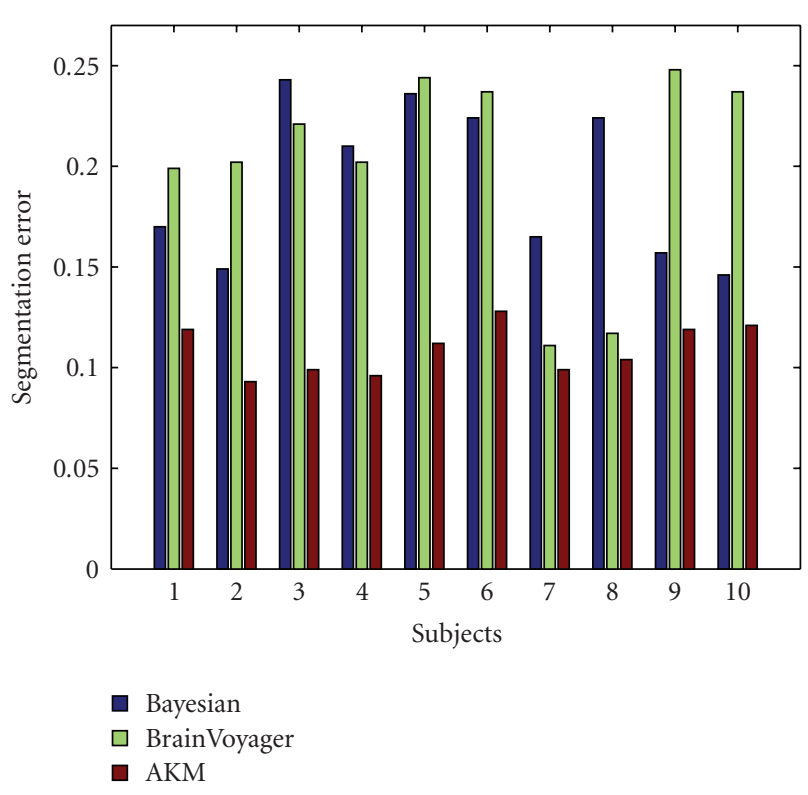

Figure 4: Classification error for Bayesian (blue), BrainVoyager (green), and AKM (red) for occipital lobes (3T).

for Bayesian and other segmentation methods $(P<.005$, Exact Wilcoxon signed rank test). Lower $L_{1}$ distances mean that AKM segmentation have more overlap with manual segmentation than other methods. Dice measures for AKM were consistently smaller than other methods.

Figures 6 and 7 explain the reason for low classification errors of AKM. Green, red, and blue curves show the intensity profile of voxels labeled as CSF, GM, and WM, respectively. The figures show how intensity histograms

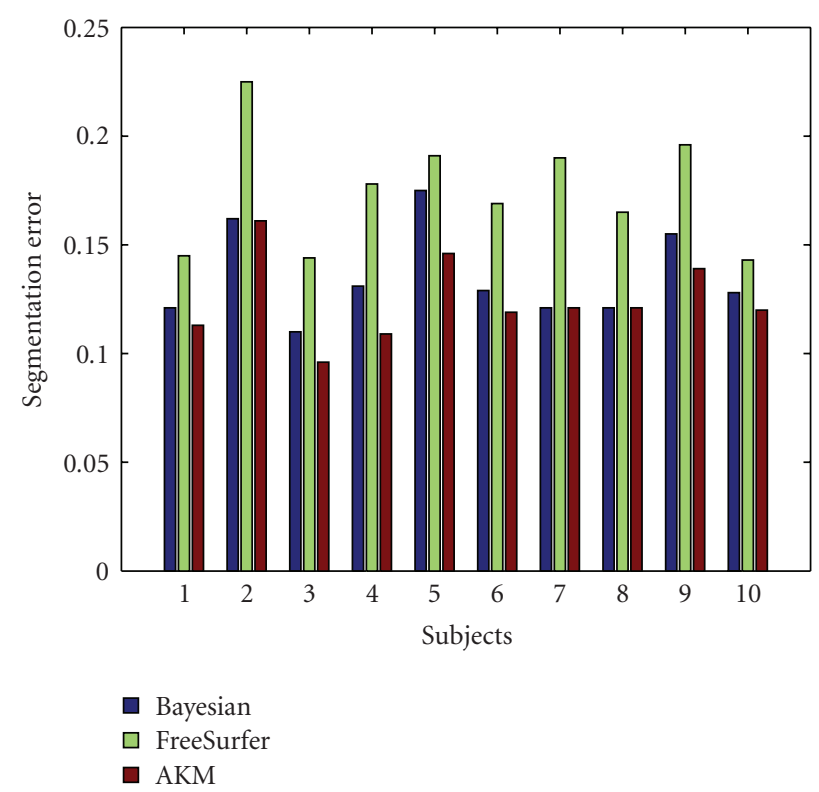

Figure 5: Classification error for Bayesian (blue), FreeSurfer (green), and AKM (red) for ten hippocampi (1.5T).

for manual segmentation voxels are similar to those for AKM segmentation. Vertical lines are threshold intensity values calculated from AKM method. Manual segmentation histograms show that each tissue type has wide range of intensities thus resulting in large overlaps between tissue types due to partial volume problems where the boundaries between tissue types are not obvious. Figure 7 shows how the large tails for each tissue types is captured by AKM yielding more accurate threshold values compared with the single Gaussian approach. Further, Table 3 and Figure 3 shows that AKM models the partial volume better than Neyman-Pearson; note that Neyman-Pearson yielded larger errors than Bayesian since the recalibration was based on the averaged thresholds. Finally, Figures 8, 9, and 10 show views of the AKM segmentation of hippocampus, prefrontal cortex, and occipital lobe subvolumes.

\section{CONCLUSION}

This paper describes an algorithm that models each tissue type in brain MRI subvolumes as a semiparametric mixture of Gaussians. The classification method which uses this algorithm results in better segmentation than a traditional, single-component Bayesian method especially when there is not enough CSF or WM in the subvolume. Human raters are good at segmenting partial volume voxels by adapting to the high variance of intensities in these regions. AKM is also able to adaptively select the bandwidth and the number of Gaussians for each tissue type. Thus, AKM approximated the manual segmentation more closely compared to Bayesian methods. AKM can automatically delineate cortical and subcortical structures which can be distinguished by intensity information. However, there are structures that cannot be segmented by intensity alone. For example, 


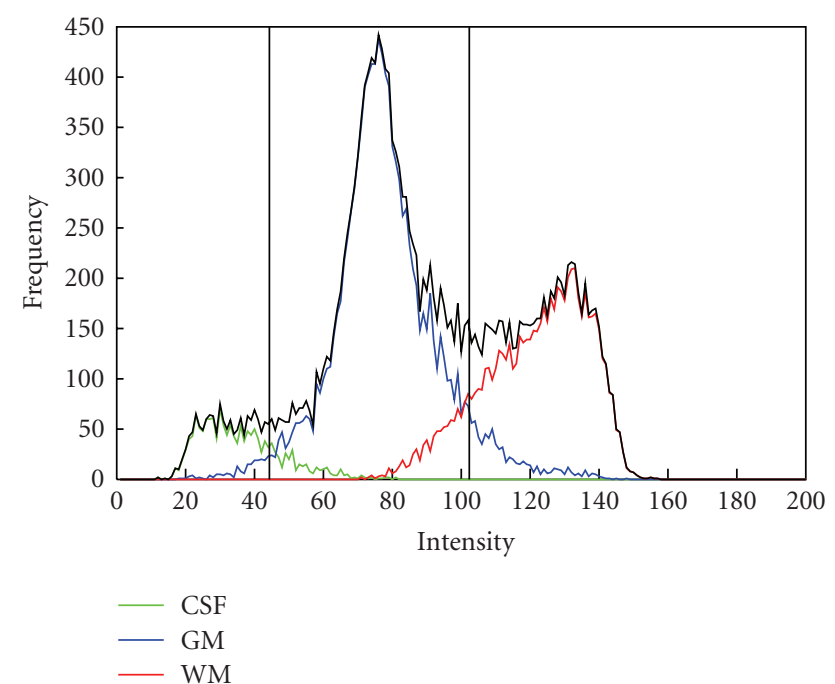

(a)

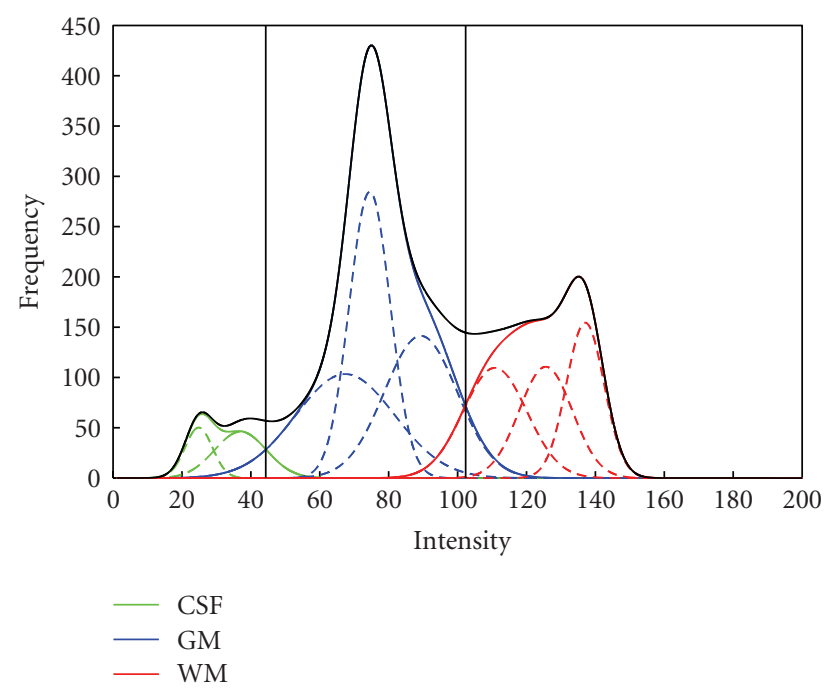

(b)

FIGURE 6: Intensity histogram for hippocampus: hand (a) and AKM (b) segmentation with vertical lines from AKM threshold. Green, blue, and red correspond to CSF, GM, and WM segmented voxels, respectively.

anterior boundary of the hippocampus merges with the amygdala which has similar intensity [39] or the anatomical boundary of prefrontal cortex and occipital lobe has to be defined with spatial information. For these structures, AKM may be useful when combined with mapping and image registration approach. Also, AKM can be applied to other imaging modalities of other anatomical structures, such as segmenting myocardium, blood, and bone in a cardiac CT scan.

\section{ACKNOWLEDGMENTS}

This work was supported by National Institute of Health Grant nos. (R01 EB00975-01, P41 RR15241-01A1, P41

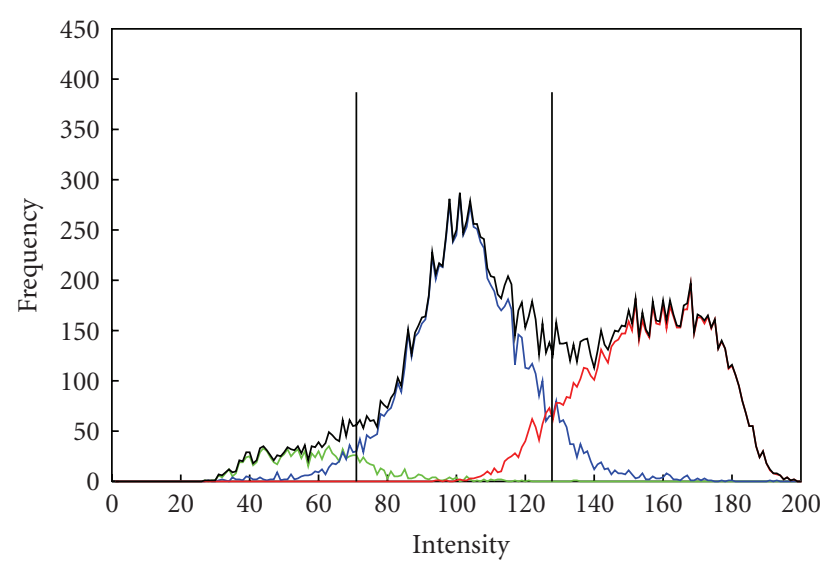

(a)

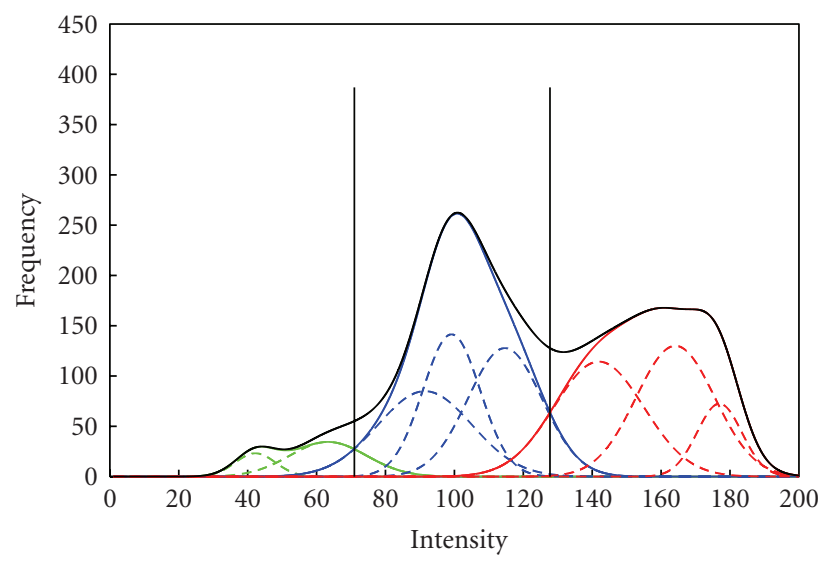

(b)

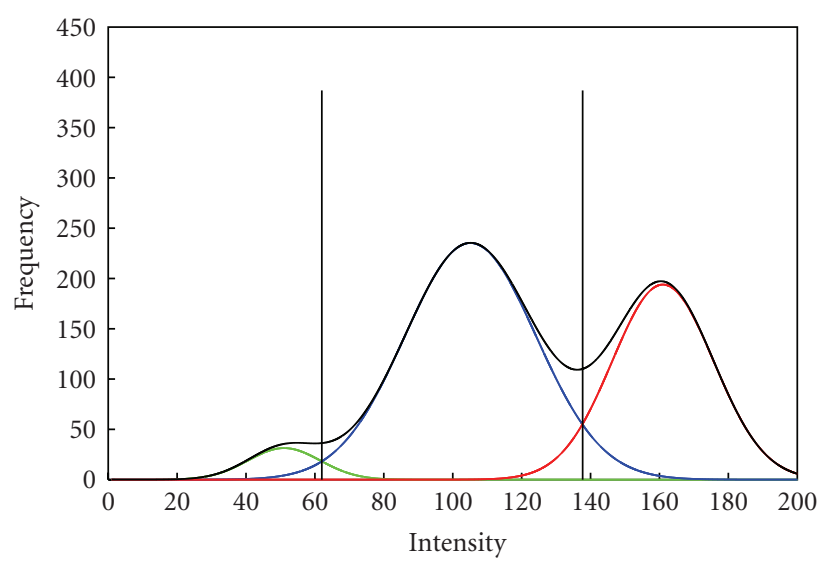

(c)

FIGURE 7: Intensity histogram for occipital lobe: hand- (a), AKM (b), and Bayesian segmentation (c) with vertical lines from AKM ((a) and (b)) and Bayesian (c).

RR15241-02S1, P50 MH071616, and P01 AG026276). Assistance from Dr. L. Wang, Dr. Y. Park, Dr. A. Qiu, Dr. S. Yantis, N. Penumetcha, and M. Hosakere is appreciated. 


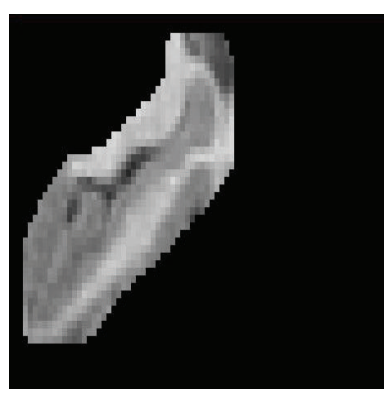

(a)

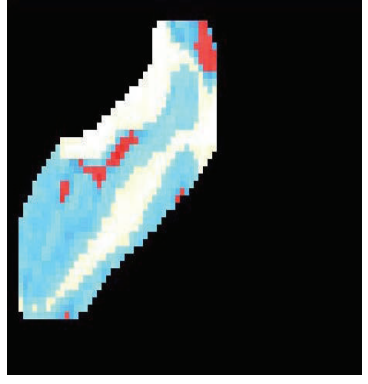

(b)
FIGURE 8: Sagittal view of left hippocampus. (a) MRI. (b) AKM segmentation (blue-GM, white-WM, red-CSF).

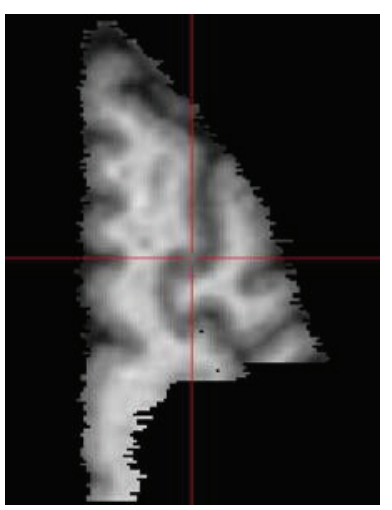

(a)

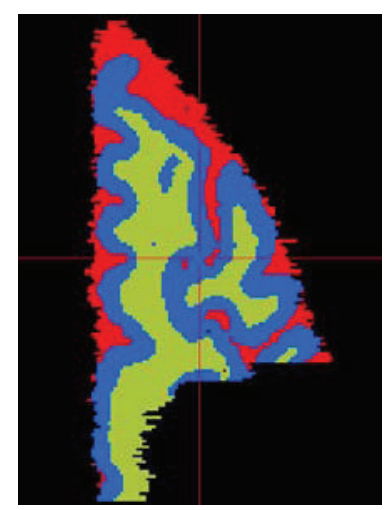

(b)
FIgUre 9: Axial view of prefrontal cortex. (a) MRI. (b) AKM segmentation (blue-GM, green-WM, red-CSF).

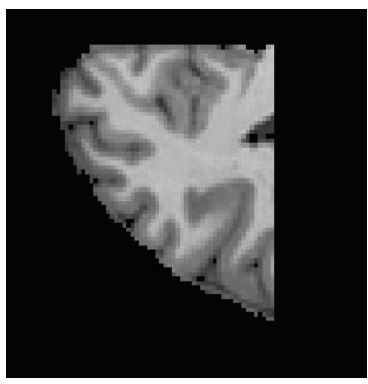

(a)

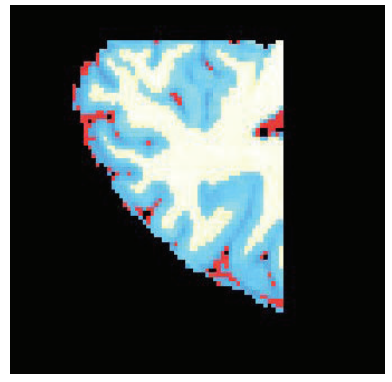

(b)
FIgure 10: Axial view of left occipital lobe. (a) MRI. (b) AKM segmentation (blue-GM, white-WM, red-CSF).

\section{REFERENCES}

[1] E. Geuze, E. Vermetten, and J. D. Bremner, "MR-based in vivo hippocampal volumetrics: 1. Review of methodologies currently employed," Molecular Psychiatry, vol. 10, no. 2, pp. 147-159, 2005.

[2] N. C. Andreasen, "Linking mind and brain in the study of mental illnesses: a project for a scientific psychopathology," Science, vol. 275, no. 5306, pp. 1586-1593, 1997.

[3] T. Onitsuka, R. W. McCarley, N. Kuroki, et al., "Occipital lobe gray matter volume in male patients with chronic schizophre- nia: a quantitative MRI study," Schizophrenia Research, vol. 92, no. 1-3, pp. 197-206, 2007.

[4] R. E. Gur, B. I. Turetsky, P. E. Cowell, et al., "Temporolimbic volume reductions in schizophrenia," Archives of General Psychiatry, vol. 57, no. 8, pp. 769-775, 2000.

[5] Y. Hirayasu, S. Tanaka, M. E. Shenton, et al., "Prefrontal gray matter volume reduction in first episode schizophrenia," Cerebral Cortex, vol. 11, no. 4, pp. 374-381, 2001.

[6] R. Kikinis, M. E. Shenton, G. Gerig, et al., "Routine quantitative analysis of brain and cerebrospinal fluid spaces with MR imaging," Journal of Magnetic Resonance Imaging, vol. 2, no. 6, pp. 619-629, 1992.

[7] J. G. Csernansky, L. Wang, D. Jones, et al., "Hippocampal deformities in schizophrenia characterized by high dimensional brain mapping," American Journal of Psychiatry, vol. 159, no. 12, pp. 2000-2006, 2002.

[8] L. Wang, D. Y. Lee, E. Bailey, et al., "Validity of largedeformation high dimensional brain mapping of the basal ganglia in adults with Tourette syndrome," Psychiatry Research, vol. 154, no. 2, pp. 181-190, 2007.

[9] S. Bouix, J. C. Pruessner, D. Louis Collins, and K. Siddiqi, "Hippocampal shape analysis using medial surfaces," NeuroImage, vol. 25, no. 4, pp. 1077-1089, 2005.

[10] J. W. Haller, A. Banerjee, G. E. Christensen, et al., "Threedimensional hippocampal MR morphometry with highdimensional transformation of a neuroanatomic atlas," Radiology, vol. 202, no. 2, pp. 504-510, 1997.

[11] O. T. Carmichael, H. A. Aizenstein, S. W. Davis, et al., "Atlasbased hippocampus segmentation in Alzheimer's disease and mild cognitive impairment," NeuroImage, vol. 27, no. 4, pp. 979-990, 2005.

[12] J. T. Ratnanather, K. N. Botteron, T. Nishino, et al., "Validating cortical surface analysis of medial prefrontal cortex," NeuroImage, vol. 14, no. 5, pp. 1058-1069, 2001.

[13] A. Qiu, M. Vaillant, P. Barta, J. T. Ratnanather, and M. I. Miller, "Surface-based Gaussian random field model with application to cortical thickness variation of left planum temporale in schizophrenia and bipolar disorder," Human Brain Mapping, vol. 29, no. 8, pp. 923-925, 2008.

[14] A. Qiu, L. Younes, L. Wang, et al., "Combining anatomical manifold information via diffeomorphic metric mappings for studying cortical thinning of the cingulate gyrus in schizophrenia," NeuroImage, vol. 37, no. 3, pp. 821-833, 2007.

[15] L. Wang, M. Hosakere, J. C. L. Trein, et al., "Abnormalities of cingulate gyrus neuroantomy in schizophrenia," Schizophrenia Research, vol. 93, no. 1-3, pp. 66-78, 2007.

[16] M. I. Miller, M. Hosakere, A. R. Barker, et al., "Labeled cortical mantle distance maps of the cingulate quantify differences between dementia of the Alzheimer type and healthy aging," Proceedings of the National Academy of Sciences of the United States of America, vol. 100, no. 25, pp. 15172-15177, 2003.

[17] B. Fischl, A. Liu, and A. M. Dale, "Automated manifold surgery: constructing geometrically accurate and topologically correct models of the human cerebral cortex," IEEE Transactions on Medical Imaging, vol. 20, no. 1, pp. 70-80, 2001.

[18] M. Joshi, J. Cui, K. Doolittle, et al., "Brain segmentation and the generation of cortical surfaces," NeuroImage, vol. 9, no. 5, pp. 461-476, 1999.

[19] M. I. Miller, A. B. Massie, J. T. Ratnanather, K. N. Botteron, and J. G. Csernansky, "Bayesian construction of geometrically based cortical thickness metrics," NeuroImage, vol. 12, no. 6, pp. 676-687, 2000. 
[20] W. M. Wells III, W. E. L. Crimson, R. Kikinis, and F. A. Jolesz, "Adaptive segmentation of MRI data," IEEE Transactions on Medical Imaging, vol. 15, no. 4, pp. 429-442, 1996.

[21] Z. Y. Shan, G. H. Yue, and J. Z. Liu, "Automated histogrambased brain segmentation in T1-weighted three-dimensional magnetic resonance head images," NeuroImage, vol. 17, no. 3, pp. 1587-1598, 2002.

[22] C. A. Cocosco, A. P. Zijdenbos, and A. C. Evans, "A fully automatic and robust brain MRI tissue classification method," Medical Image Analysis, vol. 7, no. 4, pp. 513-527, 2003.

[23] T. J. Grabowski, R. J. Frank, N. R. Szumski, C. K. Brown, and H. Damasio, "Validation of partial tissue segmentation of single-channel magnetic resonance images of the brain," NeuroImage, vol. 12, no. 6, pp. 640-656, 2000.

[24] D. W. Shattuck, S. R. Sandor-Leahy, K. A. Schaper, D. A. Rottenberg, and R. M. Leahy, "Magnetic resonance image tissue classification using a partial volume model," NeuroImage, vol. 13, no. 5, pp. 856-876, 2001.

[25] C. E. Priebe, M. I. Miller, and J. T. Ratnanather, "Segmenting magnetic resonance images via hierarchical mixture modelling," Computational Statistics \& Data Analysis, vol. 50, no. 2, pp. 551-567, 2006.

[26] C. E. Priebe and D. J. Marchette, "Alternating kernel and mixture density estimates," Computational Statistics \& Data Analysis, vol. 35, no. 1, pp. 43-65, 2000.

[27] L. F. James, C. E. Priebe, and D. J. Marchette, "Consistent estimation of mixture complexity," The Annals of Statistics, vol. 29, no. 5, pp. 1281-1296, 2001.

[28] J. T. Ratnanather, L. Wang, M. B. Nebel, et al., "Validation of semiautomated methods for quantifying cingulate cortical metrics in schizophrenia," Psychiatry Research: Neuroimaging, vol. 132, no. 1, pp. 53-68, 2004.

[29] C. B. Kirwan, C. K. Jones, M. I. Miller, and C. E. L. Stark, "High-resolution fMRI investigation of the medial temporal lobe," Human Brain Mapping, vol. 28, no. 10, pp. 959-966, 2006.

[30] A. Qiu, B. J. Rosenau, A. S. Greenberg, et al., "Estimating linear cortical magnification in human primary visual cortex via dynamic programming," NeuroImage, vol. 31, no. 1, pp. 125-138, 2006.

[31] J. P. John, L. Wang, A. J. Moffitt, H. K. Singh, M. H. Gado, and J. G. Csernansky, "Inter-rater reliability of manual segmentation of the superior, inferior and middle frontal gyri," Psychiatry Research, vol. 148, no. 2-3, pp. 151-163, 2006.

[32] R. A. Robb, D. P. Hanson, R. A. Karwoski, A. G. Larson, E. L. Workman, and M. C. Stacy, "Analyze: a comprehensive, operator-interactive software package for multidimensional medical image display and analysis," Computerized Medical Imaging and Graphics, vol. 13, no. 6, pp. 433-454, 1989.

[33] B. Fischl, D. H. Salat, E. Busa, et al., "Whole brain segmentation: automated labeling of neuroanatomical structures in the human brain," Neuron, vol. 33, no. 3, pp. 341-355, 2002.

[34] N. Kriegeskorte and R. Goebel, "An efficient algorithm for topologically correct segmentation of the cortical sheet in anatomical MR volumes," NeuroImage, vol. 14, no. 2, pp. 329346, 2001.

[35] J. T. Ratnanather, P. E. Barta, N. A. Honeycutt, et al., "Dynamic programming generation of boundaries of local coordinatized submanifolds in the neocortex: application to the planum temporale," NeuroImage, vol. 20, no. 1, pp. 359-377, 2003.

[36] T. M. Cover and J. A. Thomas, Elements of Information Theory, John Wiley \& Sons, New York, NY, USA, 1991.
[37] G. Gerig, M. Jomier, and M. Chakos, "Valmet: a new validation tool for assessing and improving 3D object segmentation," in Proceedings of the 4th International Conference on Medical Image Computing and Computer-Assisted Intervention (MICCAI '01), vol. 2208 of Lecture Notes in Computer Science, pp. 516-523, Utrecht, The Netherlands, October 2001.

[38] A. P. Zijdenbos, B. M. Dawant, R. A. Margolin, and A. C. Palmer, "Morphometric analysis of white matter lesions in MR images: method and validation," IEEE Transactions on Medical Imaging, vol. 13, no. 4, pp. 716-724, 1994.

[39] M. A. Munn, J. Alexopoulos, T. Nishino, et al., "Amygdala volume analysis in female twins with major depression," Biological Psychiatry, vol. 62, no. 5, pp. 415-422, 2007. 

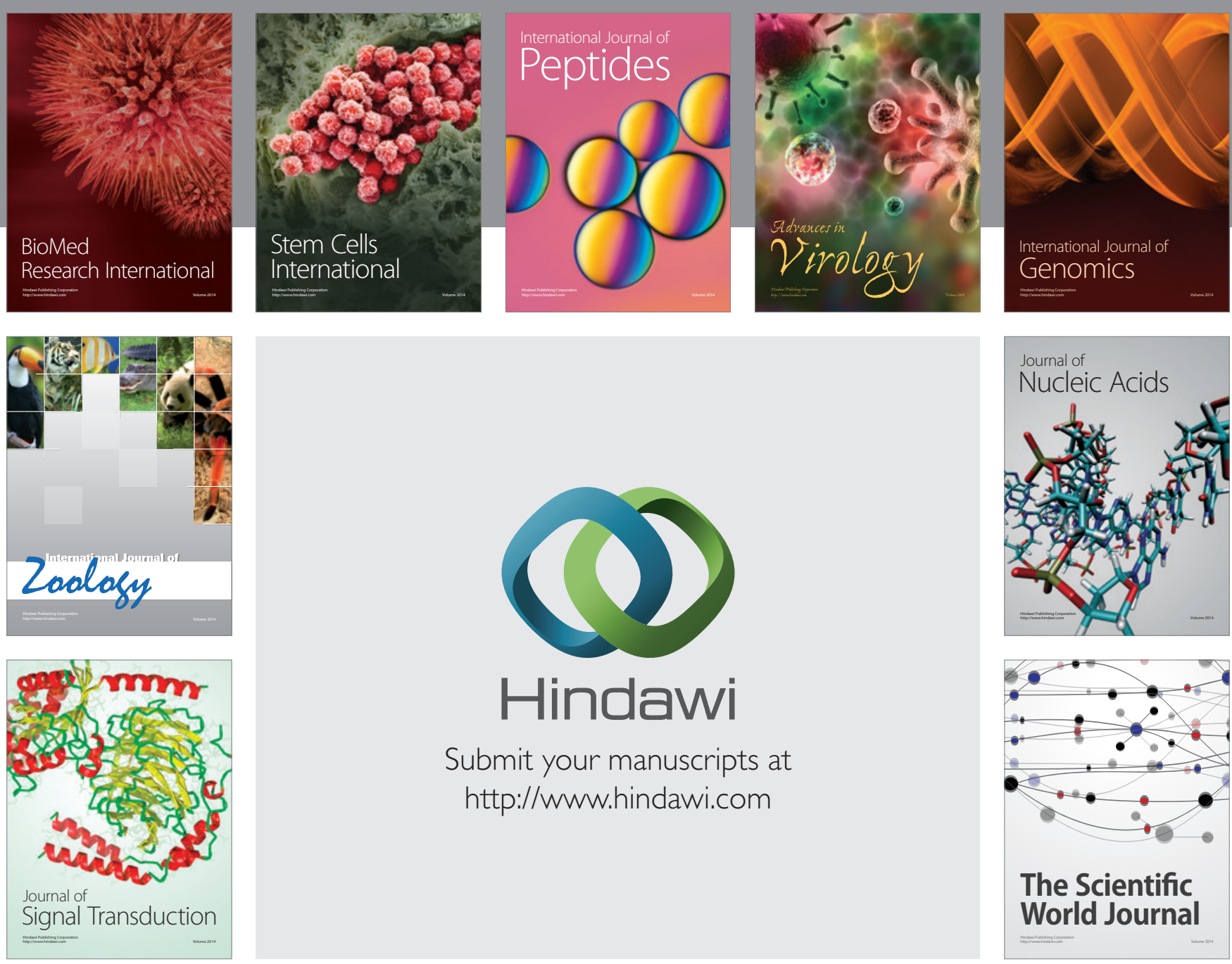

Submit your manuscripts at

http://www.hindawi.com
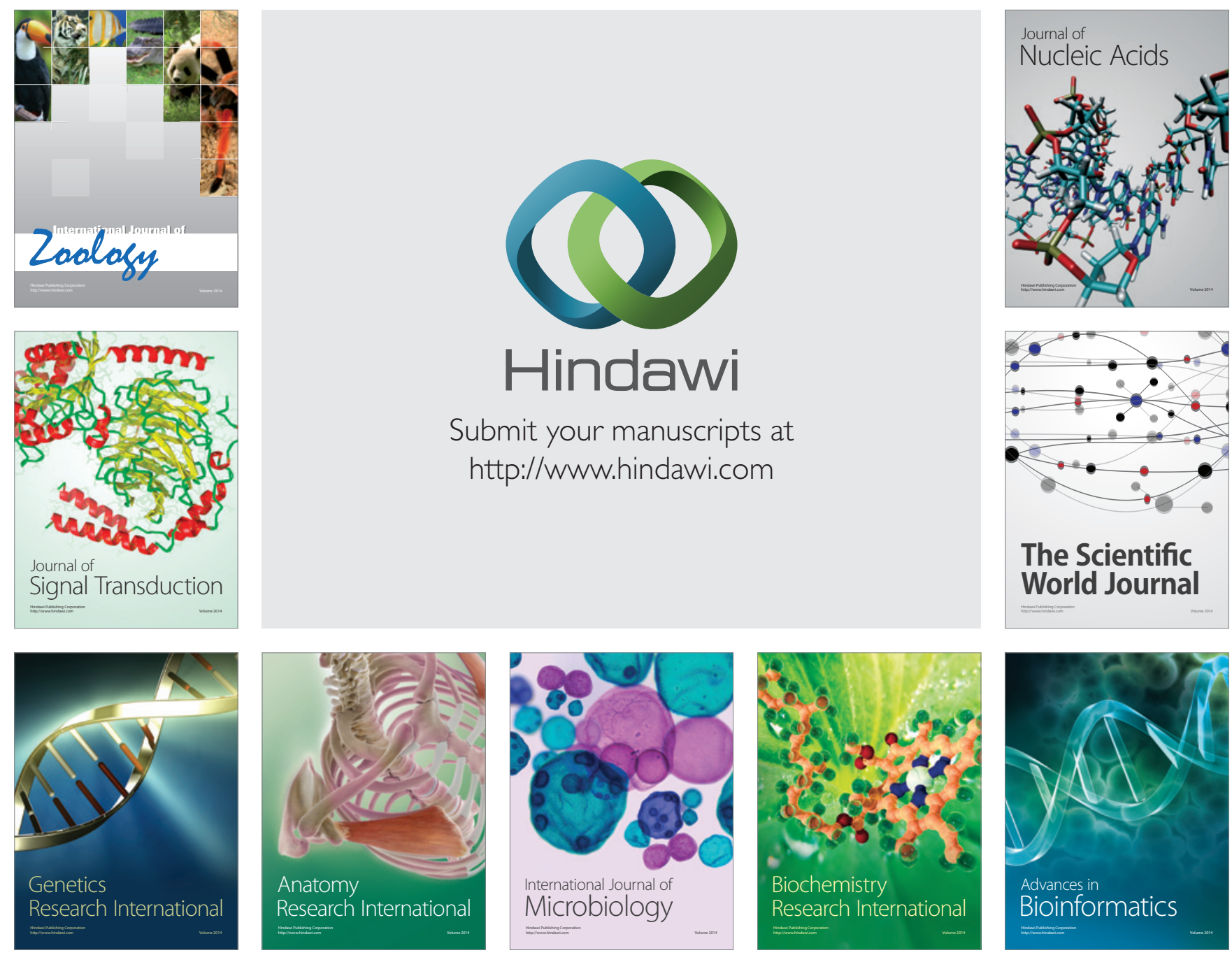

The Scientific World Journal
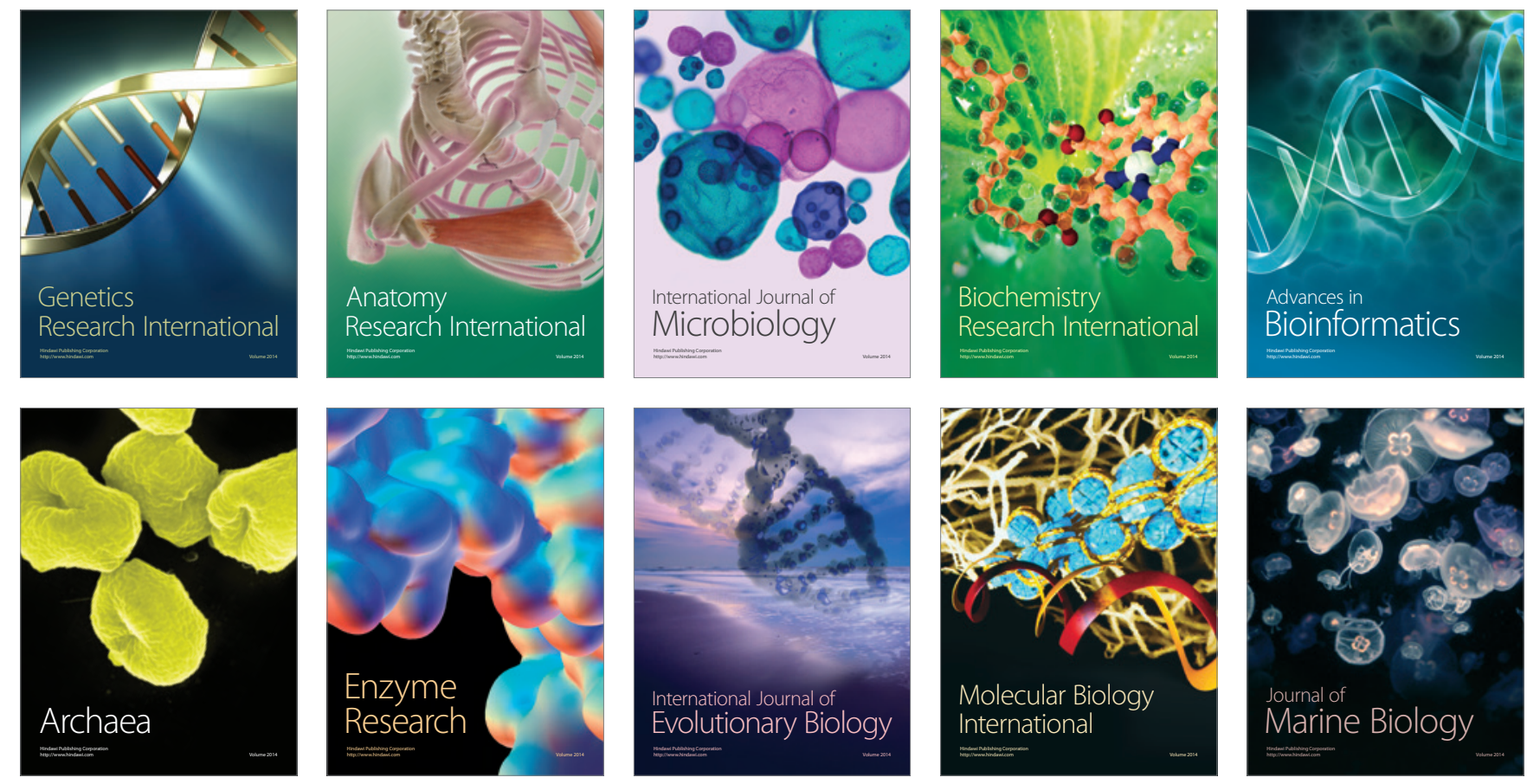